\title{
Sistematización de la gestión de la seguridad y salud ocupacional en minería
}

\author{
Occupational safety and health management system in the \\ mining industry
}

Manuel Moisés Pérez Eusebio'

Dominiotech S. A. C., Summa Tec S. A., ABC Consultores Integrales S. A. C.

\section{RESUMEN}

Objetivos: Diseñar una aplicación en plataforma SAAS (Software as a Service) que pueda ser utilizada por las empresas mineras de cualquier envergadura para ayudar a la gestión de la seguridad y salud cumpliendo con la normativa legal vigente y las políticas internas de la empresa. Métodos: Se utilizó la metodología de desarrollo de software Rational Unified Process (RUP) personalizada y certificada con ISO 9001:2000. Se utilizaron las herramientas siguientes: como lenguaje de programación, Java 2.0, Active Server Page 2.0, JavaScript, T-SQL; base de datos Microsoft, SQL Server 10.0; generador de reportes, Jasper Reports; generador de gráficos, FusionCharts 5.0; framework UI, Sencha; servidor de aplicaciones, JBoss; servidor web, IIS; sistema operativo, Microsoft Windows. Resultados: Se logró un producto simple e intuitivo que puede ser utilizado por personal minero con poco conocimiento de herramientas de software; asimismo esta aplicación permite almacenar de manera centralizada la información estructurada y no estructurada (imágenes, documentos, etc.); información disponible sin restricciones de espacio, tiempo o dispositivos; proceso simple de carga de la información a través de archivos MS Excel; gráficos estadísticos que muestran la información de una manera simple; indicadores de seguridad que ayudan a evaluar la gestión y tomar medidas preventivas a tiempo; alertas preventivas en cada proceso; emisión de los reportes requeridos por las autoridades. Conclusiones: Se ha generado una aplicación donde el ingreso y consulta de la información es muy intuitivo y rápido ya que el

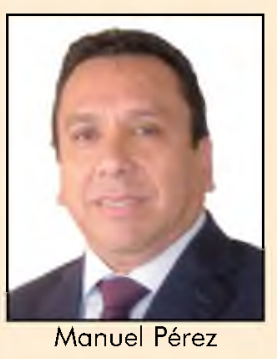

manuel.perez@dominiotech.com.pe

Historial del artículo: Recibido: 27 de agosto de 2014 Aprobado: 3 de diciembre de 2014 Disponible en línea: 30 de diciembre de 2014

personal encargado generalmente adolece de tiempos para realizar tareas de registro de información. Los procesos están muy estandarizados a nivel internacional lo cual ayuda a ser utilizada en muchos países de la región.

Palabras claves: Software, sistemas de gestión, seguridad, salud ocupacional, minería.

1 Ingeniero civil, exdocente de la Facultad de Ingeniería de Sistemas de la Universidad Nacional de Ingeniería, Lima; past president de la Asociación Peruana de Software, gerente general de Dominiotech S. A. C. 


\section{ABSTRACT}

Objectives: To design an application in SAAS (Software As A Service) platform for assisting the safety and health management in the mining companies of any size, complying with the current legal regulations and internal policies of the enterprise. Methods: It has been applied the development methodology of the Rational Unified Process (RUP) soffware customized and certified with ISO 9001:2000. The following tools were used: a programming language, Java 2.0, Active Server Page 2.0, JavaScript, T-SQL, database Microsoft SQL Server 10.0; generator of reports, Jasper Reports; originator of charts, Fusion charts 5.0; framework UI, Sencha; application server, JBoss; web server, IIS; operating system, Microsoft Windows. Results: It has been achieved a simple and an intuitive product which assists personal mining with little knowledge of software tools. In addition, this application also allows to store the structured and unstructured information in centralized manner (images, documents, etc.); available information without space restrictions; time or devices; simple process of load information through MS Excel files; statistical graphics that display the information in a simple manner; safety indicators which help to assess the management and take preventive measures in time; preventive aware in each process; and the emission of reports required by the authorities. Conclusions: From this study, it has developed an application in which the entrance and required information is very intuitive and immediate to get. Indeed, this will be useful for workers who are in charge of registering information/data and have pressure time. The processes are very standardized at international level so that they can be used in many countries.

Keywords: Software, management systems, safety, occupational health, mining.

\section{INTRODUCCIÓN}

Como antecedentes se tiene aue a nivel mundial las enfermedades profesionales siguen siendo las principales causas de las muertes relacionadas con el trabajo. Según estimaciones de la OIT (1), de un total de 2,34 millones de accidentes de trabajo mortales cada año, solo 321000 se deben a accidentes.

Los restantes 2,02 millones de muertes son causadas por diversos tipos de enfermedades relacionadas con el trabajo, lo que equivale a un promedio diario de más de 5500 muertes.

La ausencia de una prevención adecuada de las enfermedades profesionales tiene profundos efectos negativos no solo en los trabajadores y sus familias, sino también en la sociedad en su conjunto debido al enorme costo que esta genera; en particular, en lo que respecta a la pérdida de productividad y la sobrecarga de los sistemas de seguridad social.

La prevención es más eficaz y menos costosa que el tratamiento y la rehabilitación. Todos los países pueden tomar medidas concretas ahora para mejorar su capacidad para la prevención de las enfermedades profesionales y relacionadas con el trabajo. Algunos sistemas normativos son:

- La Organización Internacional del Trabajo - OIT (1) define algunos principios y lineamientos de la Seguridad y Salud en el Trabajo en los convenios 155 y 187.

- A nivel regional en la Resolución 957 (2), se define el Reglamento del Instrumento Andino de Seguridad y Salud en el Trabajo.

- A nivel Perú, la normativa Legal en Seguridad y Salud en el Trabajo está definida por la Ley 29783 (3) y la Ley $30222(4)$

- Para la actividad minera tiene el Decreto Supremo 055-2010-EM (5). Este reglamento representa la normativa más completa en seguridad y salud en el trabajo definido en el Perú. 
- Para las otras actividades se norman con el Decreto Supremo 005-2012-TR (6) y el Decreto Supremo 006-2014-TR (7)

La Superintendencia Nacional de Fiscalización Laboral (8) (SUNAFIL) tiene dentro de sus funciones la verificación del cumplimiento de las normas en cuanto a seguridad y salud en el trabajo por parte de las empresas.

Respecto al problema identificado que genera esta investigación; se inicia al identificar al sector minero como una de las actividades más riesgosa en términos de seguridad y salud de los trabajadores que la
La orientación de estas normas, reglamentos y estándares se centra en la prevención de los accidentes es por ese motivo que gran parte de los procesos y procedimientos definidos en las normas hacen hincapié en la identificación de los peligros, capacitación del personal en seguridad y salud, inspecciones y observaciones preventivas a través de procesos como IAS (Índice de Actos Seguros) o tarjetas STOPTM (Safety Training Observation Program) (14), permiten identificar potenciales riesgos y la definición de acciones preventivas y correctivas para eliminar las causas de los incidentes y accidentes.

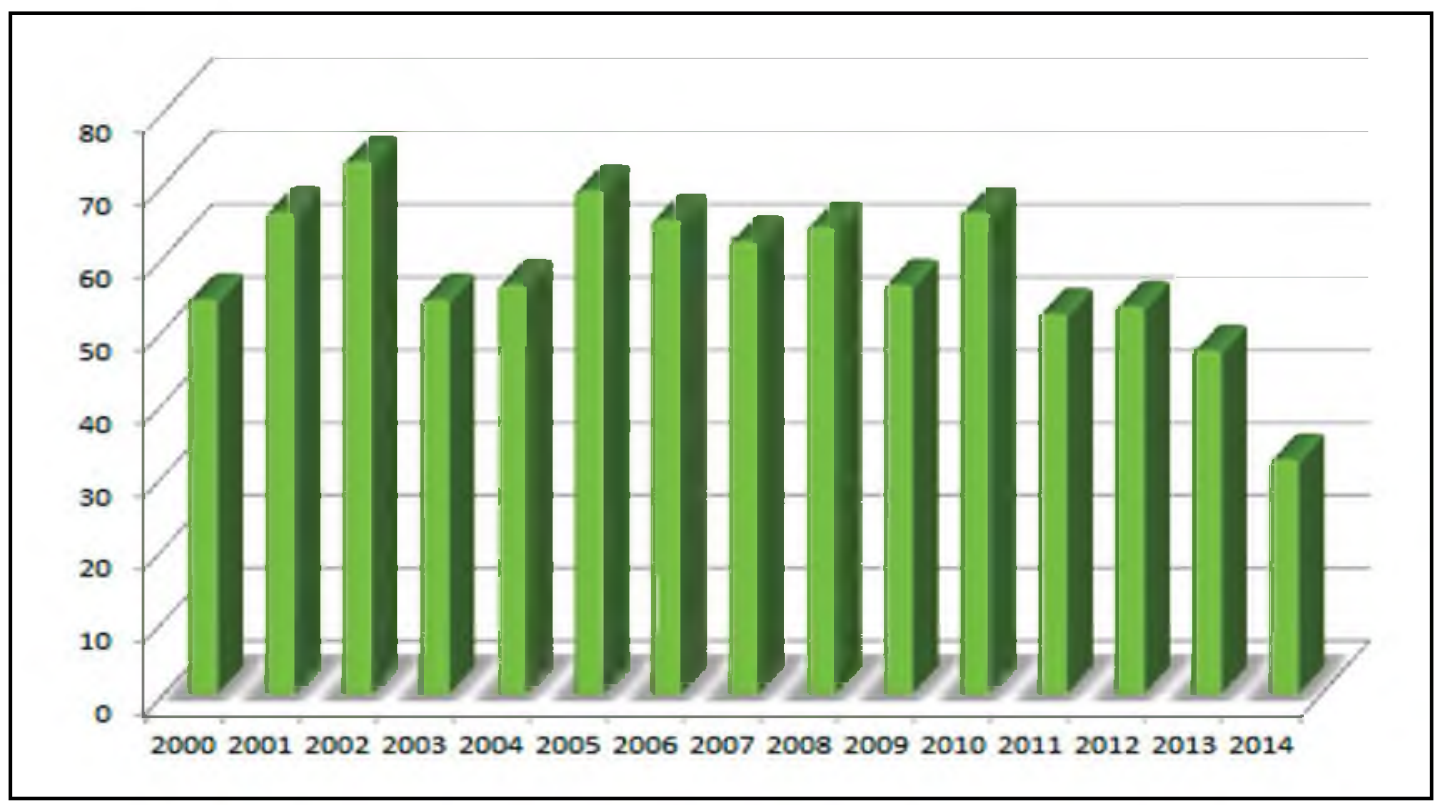

Figura $N^{\circ} 1$ : Evolución de accidentes mortales en el Perú en Minería.

Fuente: Instituto de Seguridad Minera.

realizan. Esto es debido a que las actividades tienen alta peligrosidad y se llevan a cabo en entornos muy agrestes y cambiantes.

Además de estos existen Sistema de Gestión como la OHSA 18001 (9), (Occupational Health and Safety Assessment Series, Sistemas de Gestión de Seguridad y Salud Ocupacional) que son una serie de especificaciones internacionalmente aceptadas que define los requisitos para el establecimiento, implantación y operación de un Sistema de Gestión en Seguridad y Salud Ocupacional efectivo.
De reuniones con muchos especialistas en seguridad $y$ salud en el trabajo en el sector minero se determinó que no existe un producto de software que esté en el mercado y que sea de interés al sector minero y cada empresa ha ido desarrollando soluciones independientes a cada proceso de seguridad y salud sin tener una visión integral de la solución.

Además los profesionales y trabajadores del sector no son muy proclives a usar herramientas de software ya que el gran temor es el tiempo que demoran en la carga de información. Mucha de la información es 
manejada actualmente en hojas de cálculo en donde la herramienta más común es MS Excel. Asímismo las autoridad de salud, trabajo, minas entre otros solicitan la presentación de diversos informes y reportes, los cuales se podrán generar de manera más amigable y en corto tiempo. Dichos reportes y registros listan en la Resolución Ministerial 050-2013-TR (10).

Una de las herramientas más utilizadas en la Gestión de la Seguridad y Salud en el Trabajo son el control de los indicadores de seguridad. Los indicadores son medidas cuantitativas o la información cualitativa que permite reflejar una situación determinada y la tendencia de cambios generadas en el objeto o fenómeno observado en relación con los objetivos (11).

Los indicadores ayudan a:

- Evaluar la gestión.

- Identificar oportunidades de mejoramiento.

- Adecuar a la realidad objetivos, metas y estrategias.

- Sensibilizar a las personas que toman decisiones y a quienes son objeto de las mismas.

- Tomar medidas preventivas y correctivas a tiempo.

- Comunicar ideas, pensamientos y valores de una manera resumida: "medimos lo que valoramos y valoramos lo que medimos".

Un indicador aislado, obtenido una sola vez, puede ser de poca utilidad. En cambio, cuando se analizan sus resultados a través de variables de tiempo, persona y lugar; se observan las tendencias que el mismo puede mostrar con el transcurrir del tiempo y se combina con otros indicadores apropiados, se convierten en poderosas herramientas de gestión, pues permiten mantener un diagnóstico permanentemente actualizado de la situación, tomar decisiones y verificar si éstas fueron o no acertadas.

Objetivos: El proyecto ha tenido como objetivo principal, construir una aplicación en plataforma SAAS (Software As A Service) que pueda ser utilizada por las empresas mineras de cualquier envergadura para ayudar a la gestión de la seguridad y salud cumpliendo con la normativa legal vigente y las políticas internas de la empresa.

\section{MATERIAL Y MÉTODOS}

Para el proceso de desarrollo del proyecto se utilizó una metodología de desarrollo de software basada en RUP y que la empresa personalizó y que fue certificada con ISO 9001:2000.

Proceso de implementación:

- Definición de requerimientos:

- Revisión de normativa internacional y nacional en SST

- Revisión de diversos sistemas de gestión SST : OHSAS 18001 (9), NOSA - 5 Estrellas(12), Loss Control (13) y tarjetas STOP - Dupont (14).

- Reuniones con expertos en seguridad y salud minera.

- Reuniones con gerentes de seguridad y salud de empresas mineras así como instituciones ligadas a este tema como el ISEM (Instituto de Seguridad Minera).

- Revisión de herramientas similares en el mercado.

- Elaboración de prototipo diseñado que fue validado por el mercado.

- Identificación de necesidades del usuario típico.

- Elaboración del diseño funcional.

- Codificación y pruebas unitarias de cada módulo. 
- Validación continúa de las versiones beta con los usuarios, no solo en el sector minero sino en otros sectores $y$ en otros países.

- Desarrollo de herramientas y plantillas de programación para generar pantallas y reportes de manera automática.

- Pruebas de integración y de regresión.

- Definición de los procesos de instalación e implementación del software.

- Documentación. b) Base de datos: MS SQL Server 10.0

c) Generador de reportes: Jasper Reports

d) Generador de gráficos: Fusioncharts 5.0

e) Framework UI : Sencha

f) Servidor de aplicaciones: JBoss

g) Servidor web: Internet Information Server

h) Sistema operativo: MS Windows

\section{Arquitectura general de la aplicación}
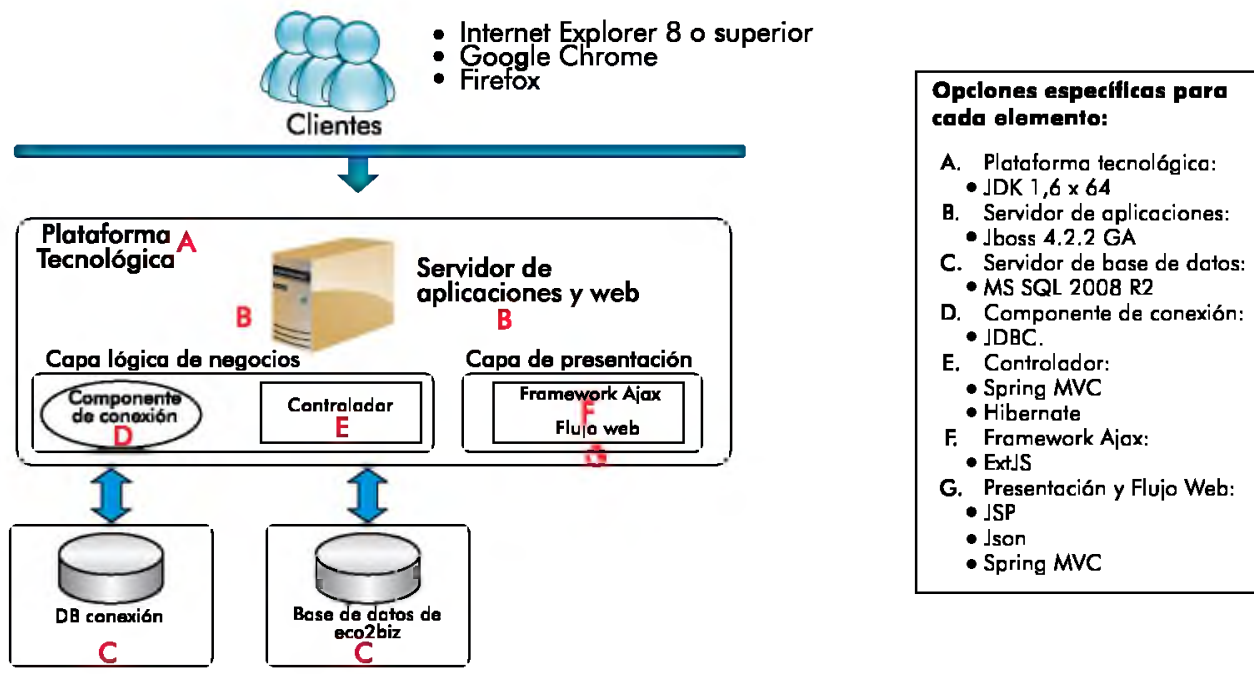

Figura $\mathrm{N}^{\circ}$ 2: Arquitectura General de la Aplicación.

- Lanzamiento del producto al mercado.

\section{Arquitectura}

La arquitectura del software se presenta en la figura $N^{\circ} 1$.

Los productos y herramientas de software que se utilizaron en la construcción del software son:

a) Lenguaje de programación: Java 2.0, Active Server Page 2.0, JavaScript, T-SQL.

\section{Interface gráfica de usuario}

La interface de la aplicación fue construida teniendo en mente los siguientes principios:

- Uso de Ajax que permita optimizar la descarga por internet.

- La interface de la aplicación tiene la forma cabecera (parte superior), menú (lateral izquierdo) y contenido (centro derecha).

- El menú está basado en el explorador de archivos de Windows (árbol jerárquico). 
- Los módulos / submódulos se presentan en forma listado / formulario.

- Los módulos / submódulos se presentan en ventanas independientes que se muestran como pestañas. Esto permite tenerlas abiertas simultáneamente.

- Los formularios podrán tener pestañas para contener información adicional.

- Las pantallas con listado tendrán funcionalidades mínima como: ordenamiento, filtrado e impresión.
El producto resultante del proyecto fue una herramienta de soffware denominada safe2biz "Software de Gestión de la Seguridad y Salud Ocupacional" que es una aplicación web implementada en arquitectura SAAS que podrá ser utilizado por empresas de cualquier sector (no solamente minería) y de cualquier país en América Latina.

Este producto fue presentado en Argentina, Colombia y Chile logrando una gran aceptación y que ha permitido que se firme un convenio con la Universidad de Belgrano para su utilización en los proyectos de SST

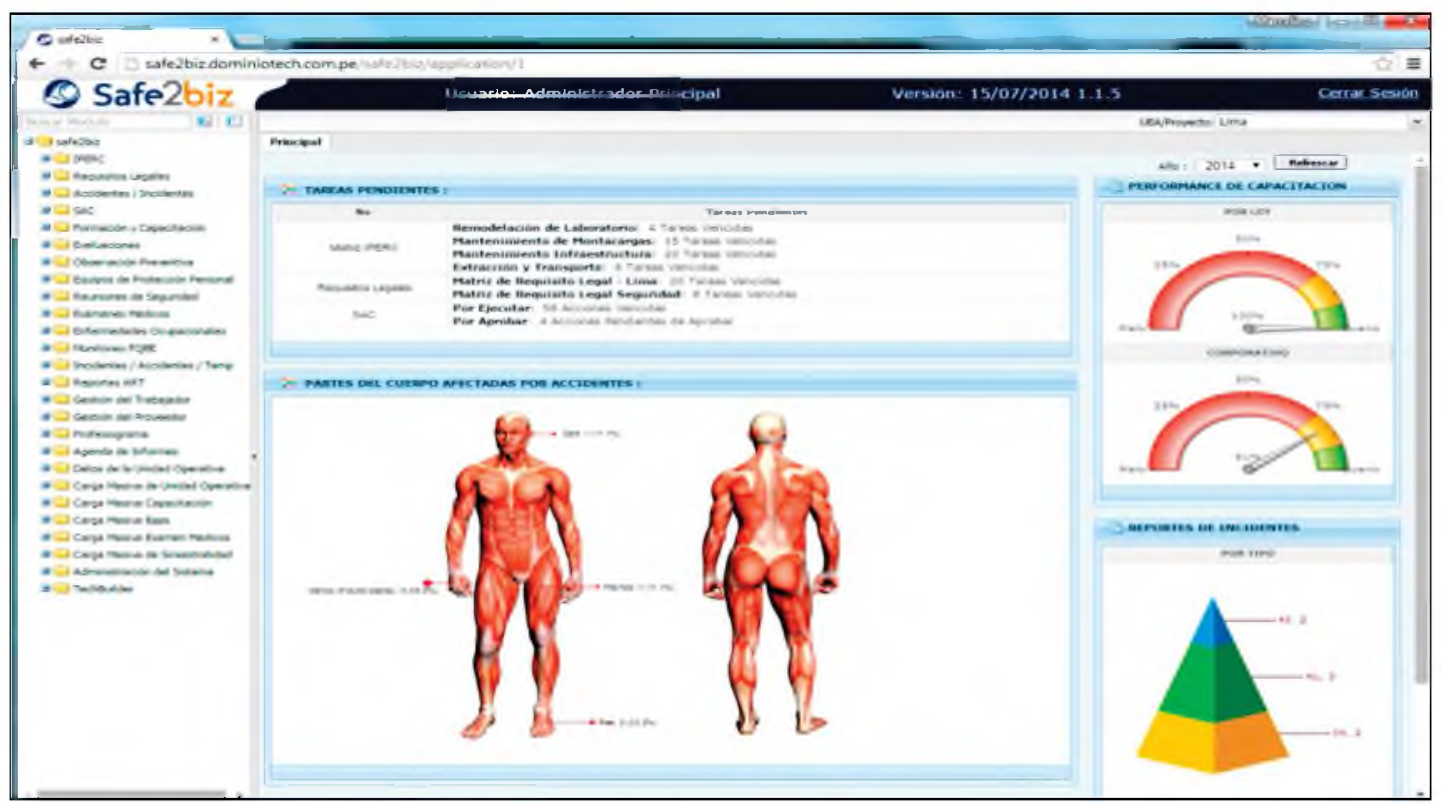

Figura $N^{\circ}$ 3: Home del Safe2iz.

Para acelerar el proceso de desarrollo se construyó un sistema que permite definir las pantallas en forma listado (grillas) y los formularios de registro de la información. También se desarrolló un patrón modelo para las pantallas de los gráficos estadísticos y los que invocan a los reportes.

\section{RESULTADOS}

El proyecto tuvo como resultado el software llamado safe2biz "Software de Gestión de la Seguridad y Salud Ocupacional" y que podrá ser utilizado no solo por la minería sino por cualquier otro sector. que la universidad realiza con diversos clientes del mercado argentino.

Los procesos que el sistema incluye son los siguientes:

- Matriz de Identificación de Peligro, Evaluación de Riesgos y Controles (IPERC).

- Gestión y Control de cumplimiento de requisitos legales.

- Registro de Accidentes / Incidentes con identificación de causas y definición de acciones de mejora. 
- Gestión de acciones de mejora de los diferentes procesos.

- Gestión, control y verificación del cumplimiento de la formación y capacitación del personal de la empresa y de los contratistas.

- Evaluaciones.

- Observación preventiva.

- Gestión y control de entrega de los equipos de protección personal.

- Reuniones de seguridad.

- Monitoreo FBQE (Físico, químico, biológico y ergonómico) de los ambientes de la empresa y del personal para la prevención de las enfermedades ocupacionales.

- Gestión del trabajador.

- Gestión del contratista.

- Profesiograma.

- Agenda de informes.

- Gestión de los exámenes médicos de los trabajadores para cumplir con los requisitos médicos necesarios para el puesto de trabajo.

- Registro y control de las enfermedades ocupacionales.

Los principales logros del producto resultante están:

- Lograr un producto muy simple e intuitivo que puede ser utilizado por personal minero con poco conocimiento de herramientas de soffware.

- Almacenar de manera centralizada la información estructurada (datos tabulares) y la data no estructurada (imágenes, documentos, etc).

- Información disponible a cualquier hora, desde cualquier lugar y usando cualquier dispositivo.

- Proceso muy simple de carga de la información a través de archivos MS Excel que es conocido por cualquier usuario.

- Gráficos estadísticos que muestran la información de una manera más simple de entender.

- Indicadores de seguridad que ayudan a evaluar la gestión y tomar medidas preventivas a tiempo.

- Alertas preventivas en cada proceso.

- Emisión de los reportes requeridos por las autoridades.

- Desarrollar un producto de software para gente que no le gusta usar software.

\section{DISCUSIÓN}

En la etapa del desarrollo del producto, se realizó un estudio de mercado de experiencias de software del mismo rubro, entre los cuales se encontraron: SCRIM, RIVO, SAEKER, INTELEX y PROSAFETY. Al realizar el estudio del software nos encontramos que muchos de estos productos solo se enfocan en los procesos de IPERC, accidentes e incidentes, evaluaciones, capacitación, entre otros; no cubriendo todos los procesos de la gestión de seguridad y salud ocupacional. Otro punto en contra que se les encontró es que sus registros se realizan de manera manual, tomando mucho tiempo en registrar la información de sus procesos.

La presentación del producto Safe2biz a diversos especialistas en seguridad de diversas industrias y países ha servido para tener una herramienta potente y útil, además ha recogido las mejores prácticas de cada una de las opiniones y sugerencias recibidas.

En base al análisis del mercado y de las reuniones realizadas con expertos en 
seguridad nace la idea de que safe 2 biz cubra todas las necesidades de los procesos de seguridad y salud ocupacional tanto para el sector minero como todas las industrias. Otro punto importante es que este tipo de proceso está muy estandarizado a nivel internacional lo cual ayuda a que este producto pueda ser utilizado en muchos países de la región.

Otra ventaja del Safe2biz tiene una herramienta amigable para el usuario, donde podrá acceder a ella, consultar de la información y realizar las tareas de registros de la información de manera rápida e intuitiva.

\section{Agradecimientos}

Al Fondo de Investigación y Desarrollo para la Competitividad (FIDECOM) por el apoyo financiero; y al CITE SOFTWARE, cuyo apoyo fue gestionado por la Asociación Peruana de Software (APESOFT).

\section{REFERENCIAS BIBLIOGRÁFICAS}

1. Organización Internacional del Trabajo [Internet]. Ginebra: OIT; [Citado el 30 de noviembre de 2014]. Seguridad y salud en el trabajo [1 pantalla]. Disponible en: http://ilo.org/global/ topics/safety-and-health-at-work/lang-es/index.htm

2. Resolución 957. Reglamento del Instrumento Andino de Seguridad y Salud en el Trabajo. Gaceta Oficial, $N^{\circ}$ 1245, (23 de septiembre de 2005).

3. Ley de Seguridad y Salud en el Trabajo. Ley $N^{\circ}$ 29783. Diario Oficial "El Peruano", (20 de agosto de 2011).

4. Ley $N^{\circ} 30222$, Ley que Modifica la Ley NN 29783. Diario Oficial "El Peruano", (11 de julio de 2014).

5. Decreto Supremo que aprueba el Reglamento de Seguridad y Salud Ocupacional y otras medidas complementarias en minería 0552010-EM. Diario Oficial "El Peruano",
(22 de agosto 2010).

6. Decreto Supremo $N^{\circ}$ 005-2012-TR. Reglamento de la Ley de $N^{\circ} 29783$ Diario Oficial "El Peruano", (Abril 2012).

7. Decreto Supremo No 006-2014-TR que modifica el Reglamento de la Ley de Seguridad y Salud en el Trabajo (en adelante, RLSST) aprobado por Decreto Supremo No D.S. 005-2012-TR. Diario Oficial "El Peruano", (9 de agosto de 2014).

8. La Superintendencia Nacional de Fiscalización Laboral. Portal "SUNAFIL" Disponible en: http://www.sunafil.gob. $\mathrm{pe} / \mathrm{portal} / \mathrm{sobre-sunafil/acerca-de-}$ sunafil

9. Asociación Española de Normalización y Certificación. OHSAS 18001:2007 Sistema de Gestión en Seguridad y Salud Ocupacional - Requisitos. Madrid: AENOR; 2007. Disponible en: https://manipulaciondealimentos. files.wordpress.com/2010/11/ ohsas-18001-2007.pdf

10. R.M. No 050-2013-TR Guía Básica sobre Sistema de Gestión de Seguridad y Salud en el Trabajo, (14 de marzo 2013).

11. RIMAC Seguros y Reaseguros [Internet]. Lima: RIMAC; [Citado el 10 de diciembre de 2014]. Indicadores de Seguridad y Salud en el Trabajo (SST) [1 pantalla]. Disponible en: http://prevencionlaboralrimac.com/ Herramientas/Indicadores-sst

12. NOSA 5 Elementos. Disponible en: https://prezi.com/d1 o7toz-u2id/ presentacion-nosa-5-estrellas/

13. Det Norske Vedtas. Manual del Control Total de Perdidas. Duluth: DNV; 2009. Disponible en: https:// clochardmoribundo.files.wordpress. com/2009/09/control-total-deperdidas.pdf

14. Tarjetas STOP - Dupont. Disponible en: http://www.latam.training.dupont. $\mathrm{com} / \mathrm{pdf} /$ free-resources/BrochureWorkshop-STOPpdf 